\title{
Physical exercise prevents motor disorders and striatal oxidative imbalance after cerebral ischemia-reperfusion
}

\author{
P.M. Sosa ${ }^{1}$, H.L. Schimidt ${ }^{2}$, C. Altermann ${ }^{1}$, A.S. Vieira ${ }^{1}$, F.W.S. Cibin ${ }^{3}$, F.P. Carpes ${ }^{2}$ \\ and P.B. Mello-Carpes ${ }^{1}$ \\ ${ }^{1}$ Grupo de Pesquisa em Fisiologia, Universidade Federal do Pampa, Uruguaiana, RS, Brasil \\ ${ }^{2}$ Grupo de Pesquisa em Neuromecânica Aplicada, Universidade Federal do Pampa, Uruguaiana, RS, Brasil \\ ${ }^{3}$ Laboratório de Biotecnologia da Reprodução, Universidade Federal do Pampa, Uruguaiana, RS, Brasil
}

\begin{abstract}
Stroke is the third most common cause of death worldwide, and most stroke survivors present some functional impairment. We assessed the striatal oxidative balance and motor alterations resulting from stroke in a rat model to investigate the neuroprotective role of physical exercise. Forty male Wistar rats were assigned to 4 groups: a) control, b) ischemia, c) physical exercise, and d) physical exercise and ischemia. Physical exercise was conducted using a treadmill for 8 weeks. Ischemia-reperfusion surgery involved transient bilateral occlusion of the common carotid arteries for $30 \mathrm{~min}$. Neuromotor performance (open-field and rotarod performance tests) and pain sensitivity were evaluated beginning at $24 \mathrm{~h}$ after the surgery. Rats were euthanized and the corpora striata was removed for assay of reactive oxygen species, lipoperoxidation activity, and antioxidant markers. Ischemia-reperfusion caused changes in motor activity. The ischemia-induced alterations observed in the open-field test were fully reversed, and those observed in the rotarod test were partially reversed, by physical exercise. Pain sensitivity was similar among all groups. Levels of reactive oxygen species and lipoperoxidation increased after ischemia; physical exercise decreased reactive oxygen species levels. None of the treatments altered the levels of antioxidant markers. In summary, ischemia-reperfusion resulted in motor impairment and altered striatal oxidative balance in this animal model, but those changes were moderated by physical exercise.
\end{abstract}

Key words: Stroke; Striatum; Locomotion; Oxidative stress; Antioxidants; Running

\section{Introduction}

Stroke is the third most common cause of death worldwide (1). Up to $20 \%$ of stroke survivors require longterm institutional care, and $15-30 \%$ are unable to perform daily life or work activities (2). Stroke events result from suppression of blood flow to the brain, which decreases oxygen and glucose delivery to brain tissue (3). This deprivation may result from disruption of a blood vessel, leading to hemorrhagic stroke, or from interruption of blood flow, leading to ischemic stroke (4). Most stroke events (85-90\%) are ischemic in origin (5). In an ischemic event, blood reperfusion leads to tissue damage (6). Such damage has deleterious effects on important cellular structures including the basal membrane and mitochondria (7).

Evidence suggests that reperfusion injury results from oxidative stress (8) characterized by increased levels of reactive oxygen species (ROS) that induce neuronal damage due to lipid peroxidation (6). Under conditions of oxidative stress, cells are unable to balance the deleterious effects of ROS through antioxidant mechanisms (8). Some brain regions, including the striatum, appear to be particularly susceptible to oxidative damage due to ROS levels (9). The striatum plays an important role in the control of voluntary movements (10) and contains a high concentration of dopaminergic receptors, which are responsible for motor activation (11). Further, dopaminergic receptors are highly susceptible to ischemic damage (12). Therefore, in models of transient ischemiareperfusion, rats can present motor impairments that may be explained by striatal damage resulting from oxidative stress and by neuronal death (13).

Correspondence: P.B. Mello-Carpes: <pamelacarpes@unipampa.edu.br>. 
The high incidence of stroke, the disabilities observed among survivors (14), and the costs of currently available treatments have promoted efforts to improve post-stroke recovery and to prevent insults to the central nervous system. One interesting strategy is physical exercise, which is easy to offer to patients and does not involve high costs. It might thus become an important public health strategy. Previous reports suggest that physical exercise may be an effective neuroprotective strategy. Aerobic exercise ameliorates memory impairment after cerebral ischemia $(12,15,16)$, reduces cognitive deficits related to aging (17), delays neurodegeneration in Alzheimer's disease models (18), and facilitates functional recovery after stroke (5). The mechanisms involved in these effects include the increase of antioxidant defenses in the hippocampus, promotion of neuronal resistance to oxidative stress (13), upregulation of BDNF (brain-derived neurotrophic factor) and VEGF (vascular endothelial growth factor) (19), and the prevention of neuronal death (1). In addition, acute exercise improves motor memory and skill acquisition (20).

Considering the results of previous studies, we assessed the neuroprotective role of physical exercise on the oxidative imbalance and motor impairments resulting from ischemia-reperfusion. Invasive experimental protocols cannot be conducted in humans, which makes animal experimentation important in advancing the understanding of behavioral and biochemical parameters associated with oxidative stress and allows dissection of brain structures. Thus, we used a rat ischemia-reperfusion model in the experiments described below.

\section{Material and Methods}

\section{Animals and experimental groups}

Forty male Wistar rats were purchased from the Central Vivarium of the Universidade Federal de Santa Maria (RS, Brazil) and housed 3 per cage under controlled light and environmental conditions (12-h light/dark cycle at $23 \pm 2^{\circ} \mathrm{C}$ and $50 \pm 10 \%$ humidity) with food and water ad libitum. All experiments were conducted in accordance with the National Institute of Health Guide for the Care and Use of Laboratory Animals (NIH, 1996) and the Animal Care and Use Committee (IRB \#0132012) of the Universidade Federal do Pampa. The weight of each rat and the liquid ingested in each cage were measured daily. At the age of 2 months, rats were randomly assigned to one of four experimental groups. A control group (SHAM) was subjected to sham surgery without occlusion of the common carotid arteries. An ischemia-reperfusion group (ISCH) was subjected to surgery to produce temporary bilateral occlusion of the common carotid arteries. An exercise group (EXERC) performed physical exercise before sham surgery. An exercise and ischemia-reperfusion group (EXERC-ISCH) performed physical exercise before ischemia-reperfusion surgery.

Rats were subjected to motor function testing beginning at $24 \mathrm{~h}$ after surgery, and 8 days after surgery. Rats were euthanized to collect brain tissue for evaluation. Figure 1 illustrates the experimental design of the study.

\section{Physical exercise protocol}

The physical exercise routine consisted of an 8-week protocol of running on a motorized treadmill built for rodents (Insight Ltda., Brazil). Running was performed at an intensity of $60-70 \%$ maximal oxygen uptake $\left(\mathrm{VO}_{2}\right)$, i.e., a treadmill belt velocity of $9-13 \mathrm{~m} / \mathrm{min}$, for $30 \mathrm{~min}$. Sessions were conducted 5 days each week at approximately the same time of day during the light time period (21). In the week before the experimental intervention, rats performed daily treadmill running for $10 \mathrm{~min}$ to habituate before performing the first $\mathrm{VO}_{2}$ test. An indirect $\mathrm{VO}_{2}$ running test was performed to determine the individual exercise intensity beginning at a low velocity and increasing by $5 \mathrm{~m} / \mathrm{min}$ every $3 \mathrm{~min}$ until the rat was unable to run. Time to fatigue ( $\mathrm{min}$ ) and the work volume $(\mathrm{m} / \mathrm{min})$ were considered as indirect measures of maximum $\mathrm{VO}_{2}$ uptake $(16,21)$. During the fourth week of exercise, an additional indirect $\mathrm{VO}_{2}$ running test was conducted to adjust the exercise intensity for each rat.

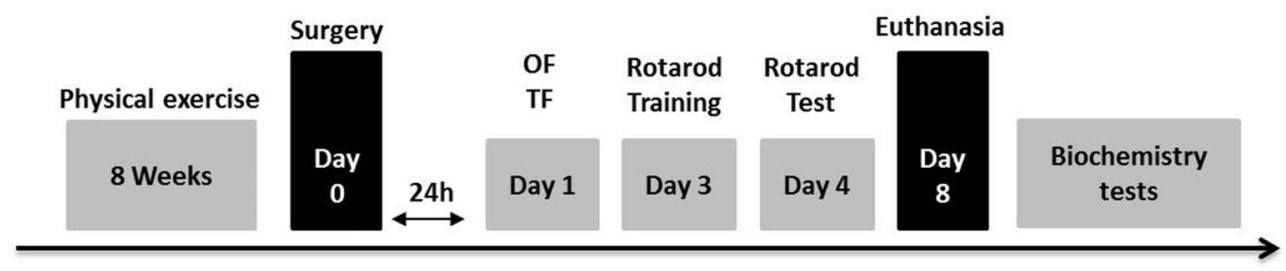

Figure 1. Rats in the exercise (EXERC) and exercise and ischemia-reperfusion groups (EXERC-ISCH) were subjected to 8 weeks of physical exercise on a treadmill for rodents. On day 0 , rats from all groups underwent surgery with or without occlusion of the carotid arteries. Twenty-four hours after surgery rats were given open-field (OF) and tail-flick tests (TF). On the third day, rats were trained in the rotarod test and on the fourth day they were given the rotarod test. To avoid changes in brain markers resulting from stress due to the rotarod test, rats were euthanized and brain tissue was collected a few days later, on the eighth day post-surgery. Bilateral striatum tissue was removed and used in subsequent biochemical assays. 


\section{Ischemia-reperfusion surgery}

After 8 weeks of intervention, rats were subjected to the ischemia-reperfusion or sham surgery procedures. The surgery was performed in the morning, under ketamine and xylazine anesthesia, 75 and $10 \mathrm{mg} / \mathrm{kg}$, respectively, given intraperitoneally. Rats were placed on a heating pad, the neck was shaved, and a midline incision was performed. The muscles and trachea were separated; the common carotid arteries were freed from the adventitial sheath and the vagus nerve, and carefully separated prior to occlusion (22). The temporary occlusion of the common carotid arteries lasted $30 \mathrm{~min}$ and was performed using a vascular clip. When restoration of blood flow in the carotid arteries was confirmed by careful observation by an experienced researcher, the neck skin incision was then closed and sutured. Body temperature was maintained during surgery, and until the rat awoke, using a heating pad. After awakening, rats were returned to their cages. Sham-operated rats underwent the same surgical procedure without application of the vascular clip.

\section{Neuromotor tasks}

Open-field test. To analyze exploratory behavior, each rat was placed in the left quadrant of a $50 \times 50 \times 39 \mathrm{~cm}$ openfield arena consisting of a wooden panel painted white and a front wall of transparent glass. Black lines were drawn on the floor to divide it into 12 equal quadrants. The number of crossings and rearings, as measures for locomotor and exploratory activity, respectively, were monitored for 5 min (23).

Rotarod test. Rats were first trained to walk on the rotarod (Insight), which was $5 \times 8 \times 20 \mathrm{~cm}$ in diameter, width and height, respectively, at a constant rotational speed of $16 \mathrm{rpm}$ for $1 \mathrm{~min}$. During training, rats were placed back on the rod each time they fell off until the session was completed. At $24 \mathrm{~h}$ after training, rats were tested on the rotarod at a constant speed of $20 \mathrm{rpm}$. Each test consisted of 5 trials lasting $60 \mathrm{~s}$ each. The time at which the rat fell off the rotarod and the number of falls were recorded. Rats that fell more than five times were excluded from the experiment and were returned to their cages (24).

Nociception evaluation. Nociception was measured using the tail-flick test (25), in which pain was induced by applying an infrared light to the rat's tail $5 \mathrm{~cm}$ from the tip. Reaction time (tail-flick latency) was measured as the interval between placing the tail on the infrared light source and its voluntary withdrawal (25).

Striatum oxidative status assessment. For tissue preparation, rats were euthanized $24 \mathrm{~h}$ after the behavioral experiments were completed. The brain was removed, and the striatum was quickly dissected and homogenized in $50 \mathrm{mM}$ Tris $\mathrm{HCl}, \mathrm{pH} 7.4(1 / 10$, w/v). The tissue samples were centrifuged at $2400 \mathrm{~g}$ for $20 \mathrm{~min}$, and supernatants (S1) were used for subsequent assays.

ROS. ROS content was assayed spectrofluorimetrically (Shimadzu model RF-5301PC, Japan) using 2',7'-dichlorofluorescein diacetate (DCFH-DA) as a probe. S1 samples were incubated in the dark with $5 \mu \mathrm{L}$ DCFH-DA ( $1 \mathrm{mM}$ ) and intracellular ROS were detected by the oxidation of DCHF-DA to fluorescent dichlorofluorescein (DCF). DCF fluorescence intensity was recorded at $520 \mathrm{~nm}$ (480 nm excitation) $30 \mathrm{~min}$ after the addition of DCFH-DA to the medium. Results are reported as $A U$ (arbitrary units).

Lipoperoxidation assay. Lipoperoxidation activity was assayed by the formation of thiobarbituric acid reactive substance (TBARS) (26). One aliquot of S1 was incubated with a $0.8 \%$ thiobarbituric acid solution in acetic acid buffer $(\mathrm{pH} 3.2)$ and $8 \%$ sodium dodecyl sulfate at $95^{\circ} \mathrm{C}$ for $2 \mathrm{~h}$, and the color reaction was measured at $532 \mathrm{~nm}$. Results are reported as nmol of malondialdehyde (MDA) per mg protein.

Antioxidant markers. Catalase (CAT) activity was determined spectrophotometrically at $240 \mathrm{~nm} \mathrm{(27)} \mathrm{by}$ monitoring $\mathrm{H}_{2} \mathrm{O}_{2}$ consumption in the presence of a $20 \mu \mathrm{L}$ sample (S1). Enzyme activity is reported in units ( $1 \mathrm{U}=1 \mu \mathrm{mol} \mathrm{H}_{2} \mathrm{O}_{2}$ decomposed/min, at $\mathrm{pH} 7$ and $25^{\circ} \mathrm{C}$ ). Glutathione ( $\mathrm{GSH}$ ) levels were determined fluorometrically (28). An aliquot of the homogenized sample was mixed (1:1) with perchloric acid $\left(\mathrm{HClO}_{4}\right)$ and centrifuged at $3000 \mathrm{~g}$ for $10 \mathrm{~min}$. After centrifugation, the protein pellet was discarded and free-SH groups were determined in the clear supernatant. An aliquot of supernatant was incubated with ortho-phthalaldehyde, and fluorescence was measured at an excitation wavelength of $350 \mathrm{~nm}$ and an emission wavelength of $420 \mathrm{~nm}$. Results are reported as $\mathrm{nmol} / \mathrm{g}$ of tissue. Superoxide dismutase (SOD) activity was measured as previously described (29) by inhibition of the auto-oxidation of epinephrine to adrenochrome. The color reaction was monitored at $480 \mathrm{~nm}$. One enzymatic unit (1 IU) was defined as the amount of enzyme necessary to inhibit the auto-oxidation rate by $50 \%$ at $26^{\circ} \mathrm{C}$.

\section{Statistical analysis}

The normality of the data distributions was verified using the Shapiro-Wilk test. Open-field and rotarod test results were compared between groups using the KruskalWallis and Dunn's post hoc tests. The Mann-Whitney test was used for further comparisons between pairs of groups. One-way analysis of variance (ANOVA) and independent $t$-tests were used to compare between-group differences in tail flick, ROS, TBARS, CAT, GSH, and SOD data. In all cases, statistical significance was set at $P<0.05$.

\section{Results}

\section{Neuromotor results}

Results of the open-field test $(P=0.001$ for crossings; $\mathrm{P}=0.01$ for rearings; Kruskal-Wallis) and the rotarod test $(P=0.03$; Kruskal-Wallis) revealed significant differences between the groups. Neuromotor deficits were observed in the rats subjected to ischemia-reperfusion surgery. In 
the open-field test, impaired performance of crossings $(P=0.048$; Figure $2 A)$ and rearings $(P=0.024$; Figure $2 B)$ were observed in the ischemia-reperfusion group compared with the sham group. Crossings $(P=0.260$; Figure $2 A)$ and rearings $(P=0.480$; Figure $2 B$ ) were similar in the physical exercise and sham groups. Physical exercise minimized the deficits resulting from ischemia-reperfusion, as shown by the crossings $(P=0.003$; Figure $2 A)$ and rearings $(P=0.004$; Figure $2 B)$ data.

Rotarod test performance was impaired in the ischemiareperfusion group compared with the sham group as shown by the number of falls $(P=0.034$; Figure $2 \mathrm{C})$ and the times at which the rats fell off the rotarod ( $P=0.038$; Figure 2D). Exercise per se did not improve performance on the rotarod test, as the number of falls $(P=0.700$; Figure $2 C$ ) and the times at which the rats fell off the rotarod observed in the physical exercise and sham groups were similar $(P=0.650$; Figure 2D). Exercise did not decrease the number of falls among rats in the ischemia-reperfusion group ( $P=0.140$; Figure $2 C$ ), but it significantly increased the latency to the first fall $(P=0.020$; Figure 2D).

\section{Nociception}

Pain sensitivity was similar among rats in the four experimental groups ( $P=0.800$; one-way ANOVA; Figure 3).

\section{Oxidative status of the striatum}

Increased oxidative stress status in the striatum was observed, as shown by the increase in ROS levels without any change in antioxidant markers after ischemia-reperfusion. Physical exercise partially reversed this condition.

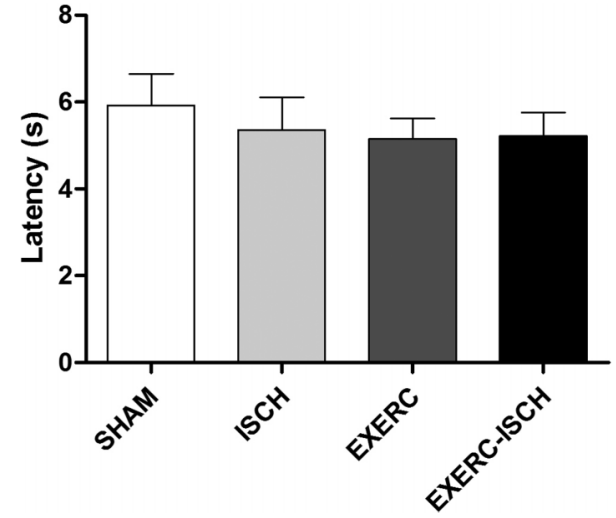

Figure 3. Transient global ischemia-reperfusion did not alter pain sensitivity measured by the tail-flick test. Data are reported as means $\pm S D$ for $n=10$ rats/group. SHAM: rats submitted to surgery without arterial occlusion; ISCH: rats submitted to ischemia-reperfusion surgery; EXERC: rats submitted to physical exercise before surgery without arterial occlusion; EXERC-ISCH: rats submitted to physical exercise before ischemia-reperfusion surgery.

Ischemia-reperfusion increased ROS $(P=0.020$; Figure $4 A)$ and TBARS ( $P=0.040$; Figure $4 B)$ in the striatum, and physical exercise reduced the increase in ROS levels $(P=0.090$; Figure 4A) but not the increase in TBARS $(P=0.250$; Figure 4B).

No significant differences in the activities of the antioxidant markers that were assayed were observed among the groups (CAT, $P=0.390 ; \mathrm{GSH}, \mathrm{P}=0.700$; SOD, $\mathrm{P}=0.340$; one-way ANOVA; Figure 5).
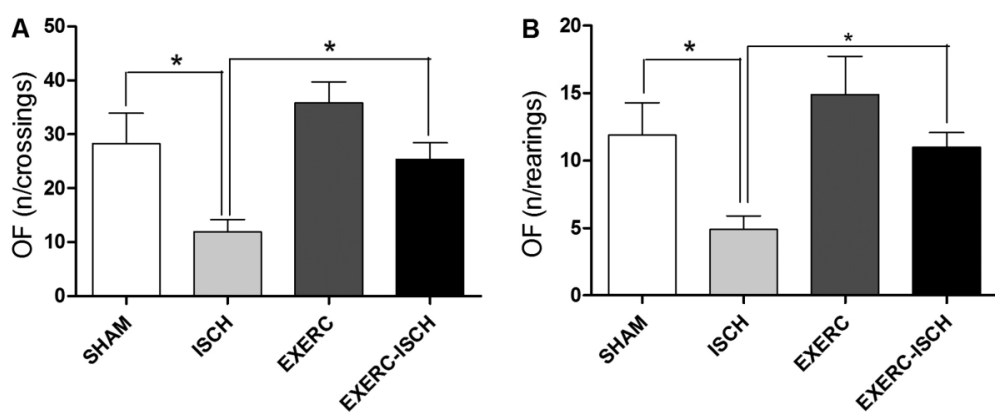

Figure 2. Transient global ischemia-reperfusion led to motor alterations and physical exercise prevented such alterations. $A$ and $B$, Results of the open-field (OF) test. The number of crossings are shown in $A$ and the number of rearings are shown in $B$. $C$ and $D$, Results of the rotarod test. The number of falls are shown in $C$ and the latency of the first fall (in seconds) is shown in $D$. Data are reported as means $\pm S D$ for $n=10$ rats/group. SHAM: rats submitted to surgery without arterial occlusion; ISCH: rats submitted to ischemia-reperfusion surgery; EXERC: rats submitted to physical exercise before surgery without arterial occlusion;
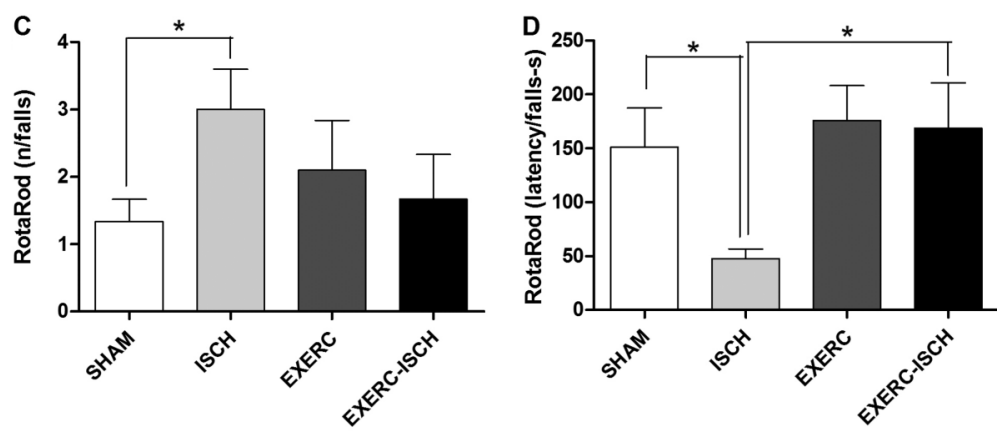
EXERC-ISCH: rats submitted to physical exercise before ischemia-reperfusion surgery. ${ }^{*} \mathrm{P}<0.05$ (Kruskal-Wallis test followed by Mann-Whitney test). 

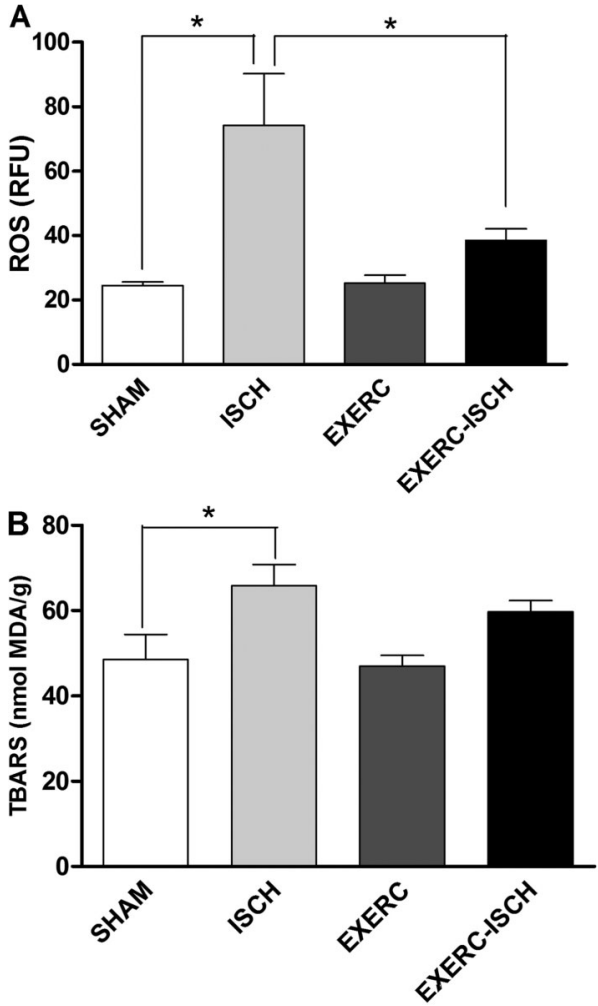

Figure 4. Transient global ischemia-reperfusion promotes an increase of reactive oxygen species and oxidative damage (lipoperoxidation) in the striatum, and physical exercise partially prevents these alterations. $A$, Reactive oxygen species (ROS) levels in the striatum and $B$, lipoperoxidation assessed by TBARS. Data are reported as means \pm SD. SHAM: rats submitted to surgery without arterial occlusion; $\mathrm{ISCH}$ : rats submitted to ischemia-reperfusion surgery; EXERC: rats submitted to physical exercise before surgery without arterial occlusion; EXERC-ISCH: rats submitted to physical exercise before ischemia-reperfusion surgery. ${ }^{*} \mathrm{P}<0.05$ (one-way ANOVA; $t$-test).

\section{Discussion}

We assessed the neuroprotective role of physical exercise on striatal oxidative balance and motor impairments resulting from ischemia-reperfusion injury in rats. Our results indicated that ischemia-reperfusion led to significant neuromotor impairments without changing nociception. Animals subjected to ischemia-reperfusion also experienced oxidative stress resulting in oxidative imbalance in the striatum. The damage observed in the striatum is consistent with the neuromotor deficits observed in the ischemia-reperfusion group (9). In this study, neuromotor impairment was demonstrated by the results of the rotarod and open-field tests.

Among our main findings is the capability of physical exercise to protect, although partially, against impairments resulting from an ischemia-reperfusion insult. Physical exercise reversed the impairments in the rotarod test (i.e., time to the first fall) and open-field test (i.e., crossings and rearings) performance observed in the ischemia-reperfusion group. Ischemia-reperfusion is known to induce the deficits in motor development and balance that are measured by the rotarod test (30) and has also been associated with lower neuronal density and area in the striatum after the ischemia-reperfusion injury (30). A neuroprotective role of exercise performed for 14 days before ischemia has been reported in rats, with similar effects in rats trained after ischemia (5).

Nociception was not affected by the ischemic event, which may be explained by the fact that nociception does not depend on striatal activity, but by nociception-specific cortical regions, areas were not evaluated in this study. Those cortical regions are also known to be less sensitive to ischemic events than the hippocampus and striatum are (31). The lack of change in nociception supports a model in which the motor impairments we observed resulted from damage to the striatum.

The motor impairments observed here most likely resulted from the ischemic event that the rats experienced (16). Neuronal degeneration induced by ischemia-reperfusion
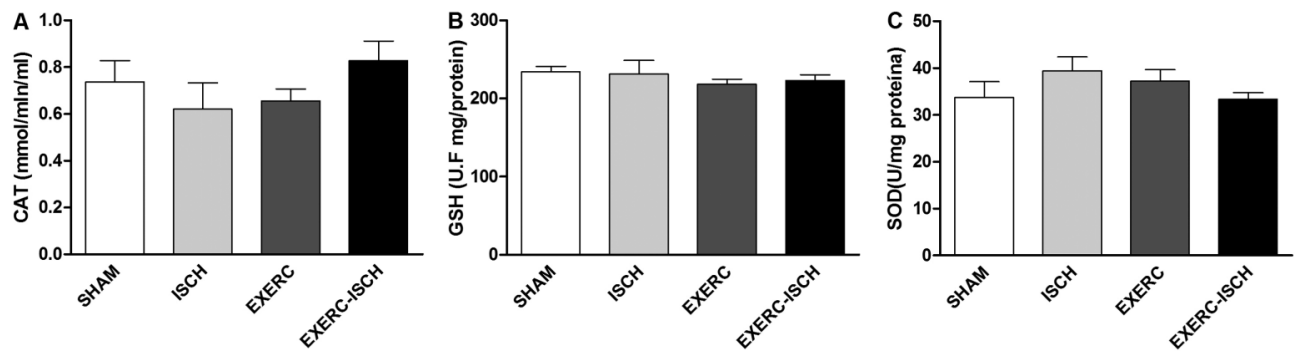

Figure 5. Transient global ischemia-reperfusion and physical exercise did not alter antioxidant markers. $A$, catalase activity (CAT), $B$, glutathione (GSH) levels, and $C$, superoxide dismutase activity (SOD) in the four groups. Data are reported as means \pm SD. SHAM: rats submitted to surgery without arterial occlusion; ISCH: rats submitted to ischemia-reperfusion surgery; EXERC: rats submitted to physical exercise before surgery without arterial occlusion; EXERC-ISCH: rats submitted to physical exercise before ischemiareperfusion surgery. There were no significant differences among groups (one-way ANOVA; $t$-test). 
is associated with conditions of oxidative stress resulting from high levels of fatty acids in the brain (6). The striatum is one of the brain regions most affected by oxidative stress in ischemia-reperfusion and its relatively high densities of GABA receptors and glutamatergic neurons may be related to this neurotoxicity (12). Oxidative stress plays a major role in various pathological conditions, and it may occur in the striatum during aging (17), chronic unpredictable stress situations (32), neurodegenerative diseases such as Alzheimer's and Parkinson's diseases (18,33), and also after strokes (16). Oxidative imbalance in the striatum is related to the loss of dopaminergic neurons and neurotoxicity $(12,34)$ and to damage to DNA/RNA, lipids and proteins, resulting in altered cellular and molecular function and increased cell death $(6,8,35,36)$.

The motor impairments observed here appear to be related to increased levels of ROS and lipoperoxidation in the striatum, leading to oxidative imbalance in this brain region (34). We found that exercise was effective for avoiding motor impairments and that it decreased ROS levels but not lipoperoxidation activity. A recent evaluation of the neuroprotective role of exercise in ischemiareperfusion injury reported similar results for lipoperoxidation (16). The effects of exercise may be mediated by mitochondrial biogenesis; edema reduction, which would improve blood flow in the ischemic region; and the attenuation of acute neurotoxicity, which would facilitate the reorganization of the injured brain tissue $(12,13,37,38)$.

\section{References}

1. Park S, Kim da S, Kang S. Exercise training attenuates cerebral ischemic hyperglycemia by improving hepatic insulin signaling and beta-cell survival. Life Sci 2013; 93: 153-160, doi: 10.1016/j.lfs.2013.06.005.

2. Chen S, Yin ZJ, Jiang C, Ma ZQ, Fu Q, Qu R, et al. Asiaticoside attenuates memory impairment induced by transient cerebral ischemia-reperfusion in mice through anti-inflammatory mechanism. Pharmacol Biochem Behav 2014; 122: 7-15, doi: 10.1016/j.pbb.2014.03.004.

3. Zamani M, Katebi M, Mehdizadeh M, Kafami L, Soleimani M. Combination therapy with $\mathrm{A} 1$ receptor agonist and vitamin $\mathrm{C}$ improved working memory in a mouse model of global ischemia-reperfusion. Basic Clin Neurosci 2013; 4: 111-116.

4. Mestriner RG, Miguel PM, Bagatini PB, Saur L, Boisserand LS, Baptista PP, et al. Behavior outcome after ischemic and hemorrhagic stroke, with similar brain damage, in rats. Behav Brain Res 2013; 244: 82-89, doi: 10.1016/j.bbr.2013. 02.001.

5. Heo M, Kim E. Beneficial effects of antecedent exercise training on limb motor function and calpain expression in a rat model of stroke. J Phys Ther Sci 2013; 25: 943-946, doi: 10.1589/jpts.25.943.

6. Allen $\mathrm{CL}$, Bayraktutan U. Oxidative stress and its role in the pathogenesis of ischaemic stroke. Int $J$ Stroke 2009; 4: 461-470, doi: 10.1111/j.1747-4949.2009.00387.x.

7. Gulati P, Singh N. Neuroprotective effect of tadalafil, a PDE5 inhibitor, and its modulation by L-NAME in mouse model of
As expected, levels of antioxidant enzymes (CAT and SOD) and GSH, a key non-enzymatic antioxidant, were not changed by exercise (37). Cechetti et al. (16) reported similar results in the cortex and hippocampus.

Understanding the mechanisms of brain injury, the brain's defense responses, and the adaptations in response to long-term exercise is important for improving the strategies for rehabilitation after ischemic events. Our research supports a model in which physical exercise reverses deficits in locomotor behavior and striatal oxidative balance but does not improve antioxidant status. We found that a relatively short period of physical conditioning benefited animals subjected to ischemia-reperfusion surgery.

In summary, our results demonstrate that physical exercise performed during 8 weeks before ischemiareperfusion was effective to avoid or minimize motor deficits and oxidative stress conditions in the striatum. In this animal model, exercise was neuroprotective, attenuating the severity of ischemia-reperfusion sequelae.

\section{Acknowledgments}

This research was supported by CAPES (PROCADCAPES/2013 \#88881.068493/2014-01), CNPq (Universal/2014 \#441548/2014-8) and Universidade Federal do Pampa. H.L. Shimidt and C. Altermann received master fellowships from CAPES. A.S. Vieira received a student fellowship from FAPERGS. ischemia-reperfusion injury. J Surg Res 2014; 186: 475-483, doi: $10.1016 /$ j.jss.2013.08.005.

8. Abramov AY, Scorziello A, Duchen MR. Three distinct mechanisms generate oxygen free radicals in neurons and contribute to cell death during anoxia and reoxygenation. J Neurosci 2007; 27: 1129-1138, doi: 10.1523/JNEUROSCI. 4468-06.2007.

9. Li PF, Zhan HQ, Li SY, Liu RL, Yan FL, Cui TZ, et al. Lactuside $B$ decreases aquaporin- 4 and caspase- 3 mRNA expression in the hippocampus and striatum following cerebral ischaemia-reperfusion injury in rats. Exp Ther Med 2014; 7: 675-680.

10. Baez-Mendoza R, Schultz W. The role of the striatum in social behavior. Front Neurosci 2013; 7: 233.

11. Teixeira AM, Reckziegel P, Muller L, Pereira RP, Roos DH, Rocha JB, et al. Intense exercise potentiates oxidative stress in striatum of reserpine-treated animals. Pharmacol Biochem Behav 2009; 92: 231-235, doi: 10.1016/j.pbb. 2008.11.015

12. Park CY, Lee SH, Kim BK, Shin MS, Kim CJ, Kim H. Treadmill exercise ameliorates impairment of spatial learning ability through enhancing dopamine expression in hypoxic ischemia brain injury in neonatal rats. J Exerc Rehabil 2013; 9: 406-412, doi: 10.12965/jer.130053.

13. Lu F, Nakamura T, Toyoshima T, Liu Y, Hirooka K, Kawai N, et al. Edaravone, a free radical scavenger, attenuates behavioral deficits following transient forebrain ischemia by 
inhibiting oxidative damage in gerbils. Neurosci Lett 2012; 506: 28-32, doi: 10.1016/j.neulet.2011.10.041.

14. Zheng G, Chen B, Fang Q, Yi H, Lin Q, Chen L, et al. Primary prevention for risk factors of ischemic stroke with Baduanjin exercise intervention in the community elder population: study protocol for a randomized controlled trial. Trials 2014; 15: 113, doi: 10.1186/1745-6215-15-113.

15. Schimidt HL, Vieira A, Altermann C, Martins A, Sosa P, Santos FW, et al. Memory deficits and oxidative stress in cerebral ischemia-reperfusion: neuroprotective role of physical exercise and green tea supplementation. Neurobiol Learn Mem 2014; 114: 242-250, doi: 10.1016/j.nIm.2014. 07.005 .

16. Cechetti F, Worm PV, Elsner VR, Bertoldi K, Sanches E, Ben $\mathrm{J}$, et al. Forced treadmill exercise prevents oxidative stress and memory deficits following chronic cerebral hypoperfusion in the rat. Neurobiol Learn Mem 2012; 97: 90-96, doi: 10.1016/j.nlm.2011.09.008.

17. Flores MF, Martins A, Schimidt HL, Santos FW, Izquierdo I, Mello-Carpes PB, et al. Effects of green tea and physical exercise on memory impairments associated with aging. Neurochem Int 2014; 78: 53-60, doi: 10.1016/j.neuint. 2014.08.008.

18. Lin TW, Shih YH, Chen SJ, Lien CH, Chang CY, Huang TY, et al. Running exercise delays neurodegeneration in amygdala and hippocampus of Alzheimer's disease (APP/ PS1) transgenic mice. Neurobiol Learn Mem 2015; 118: 189-197, doi: 10.1016/j.nlm.2014.12.005.

19. Uysal N, Kiray M, Sisman AR, Camsari UM, Gencoglu C, Baykara B, et al. Effects of voluntary and involuntary exercise on cognitive functions, and VEGF and BDNF levels in adolescent rats. Biotech Histochem 2015; 90: 55-68, doi: 10.3109/10520295.2014.946968.

20. Skriver K, Roig M, Lundbye-Jensen J, Pingel J, Helge JW, Kiens B, et al. Acute exercise improves motor memory: exploring potential biomarkers. Neurobiol Learn Mem 2014; 116: 46-58, doi: 10.1016/j.nlm.2014.08.004.

21. Brooks GA, White TP. Determination of metabolic and heart rate responses of rats to treadmill exercise. J Appl Physiol Respir Environ Exerc Physiol 1978; 45: 1009-1015.

22. Collino M, Aragno M, Mastrocola R, Gallicchio M, Rosa AC, Dianzani $C$, et al. Modulation of the oxidative stress and inflammatory response by PPAR-gamma agonists in the hippocampus of rats exposed to cerebral ischemia/reperfusion. Eur J Pharmacol 2006; 530: 70-80, doi: 10.1016/ j.ejphar.2005.11.049.

23. Bonini JS, Bevilaqua LR, Zinn CG, Kerr DS, Medina JH, Izquierdo I, et al. Angiotensin II disrupts inhibitory avoidance memory retrieval. Horm Behav 2006; 50: 308-313, doi: 10.1016/j.yhbeh.2006.03.016.

24. de Castro BM, Pereira GS, Magalhaes V, Rossato JI, De Jaeger X, Martins-Silva C, et al. Reduced expression of the vesicular acetylcholine transporter causes learning deficits in mice. Genes Brain Behav 2009; 8: 23-35, doi: 10.1111/ j.1601-183X.2008.00439.x.

25. Tjølsen A, Lund A, Berge OG, Hole K. An improved method for tail-flick testing with adjustment for tail-skin temperature. J Neurosci Methods 1989; 26: 259-265, doi: 10.1016/01650270(89)90124-6.
26. Ohkawa H, Ohishi N, Yagi K. Assay for lipid peroxides in animal tissues by thiobarbituric acid reaction. Anal Biochem 1979; 95: 351-358, doi: 10.1016/0003-2697(79)90738-3.

27. Aebi H. Catalase in vitro. Methods Enzymol 1984; 105: 121126.

28. Hissin PJ, Hilf R. A fluorometric method for determination of oxidized and reduced glutathione in tissues. Anal Biochem 1976; 74: 214-226, doi: 10.1016/0003-2697(76)90326-2.

29. Misra KB. Reliability optimization of a series-parallel system. IEEE Trans Reliability 1972; 21: 230-238, doi: 10.1109/ TR.1972.5216000.

30. de Araujo FL, Bertolino G, Goncalves RB, Marini LC, Coimbra NC, de Araujo JE. Neuropathology and behavioral impairments after three types of global ischemia surgery in Meriones unguiculatus: evidence in motor cortex, hippocampal CA1 region and the neostriatum. J Neurol Sci 2012; 312: 73-78, doi: 10.1016/j.jns.2011.08.019.

31. Pereira LO, Nabinger PM, Strapasson AC, Nardin P, Goncalves CA, Siqueira IR, et al. Long-term effects of environmental stimulation following hypoxia-ischemia on the oxidative state and BDNF levels in rat hippocampus and frontal cortex. Brain Res 2009; 1247: 188-195, doi: 10.1016/ j.brainres.2008.10.017.

32. Che Y, Zhou Z, Shu Y, Zhai C, Zhu Y, Gong S, et al. Chronic unpredictable stress impairs endogenous antioxidant defense in rat brain. Neurosci Lett 2015; 584: 208-213, doi: 10.1016/j.neulet.2014.10.031.

33. Le Douaron G, Schmidt F, Amar M, Kadar H, Debortoli L, Latini A, et al. Neuroprotective effects of a brain permeant 6aminoquinoxaline derivative in cell culture conditions that model the loss of dopaminergic neurons in Parkinson disease. Eur J Med Chem 2015; 89: 467-479, doi: 10.1016/j. ejmech.2014.10.067.

34. Masoud ST, Vecchio LM, Bergeron Y, Hossain MM, Nguyen LT, Bermejo MK, et al. Increased expression of the dopamine transporter leads to loss of dopamine neurons, oxidative stress and I-DOPA reversible motor deficits. Neurobiol Dis 2015; 74: 66-75, doi: 10.1016/j.nbd.2014.10.016.

35. Colamartino M, Santoro M, Duranti G, Sabatini S, Ceci R, Testa A, et al. Evaluation of levodopa and carbidopa antioxidant activity in normal human lymphocytes in vitro: implication for oxidative stress in Parkinson's disease. Neurotox Res 2015; 27: 106-117, doi: 10.1007/s12640014-9495-7.

36. Lalith Kumar V, Muralidhara. Ameliorative effects of ferulic Acid against lead acetate-induced oxidative stress, mitochondrial dysfunctions and toxicity in prepubertal rat brain. Neurochem Res 2014; 39: 2501-2515, doi: 10.1007/s11064014-1451-7.

37. Marques-Aleixo I, Oliveira PJ, Moreira PI, Magalhaes J, Ascensao A. Physical exercise as a possible strategy for brain protection: evidence from mitochondrial-mediated mechanisms. Prog Neurobiol 2012; 99: 149-162, doi: 10.1016/j.pneurobio.2012.08.002.

38. Wang Z, Myers KG, Guo Y, Ocampo MA, Pang RD, Jakowec MW, et al. Functional reorganization of motor and limbic circuits after exercise training in a rat model of bilateral parkinsonism. PLoS One 2013; 8: e80058, doi: 10.1371/journal.pone.0080058. 